\title{
Impact of Ivabradine in Decompensated Heart Failure related to Chemotherapy-induced Cardiomyopathy
}

\author{
YUSUKE NAKANO ${ }^{1}$, HIROHIKO ANDO ${ }^{1}$, WATARU SUZUKI ${ }^{1}$, HIROFUMI OHASHI ${ }^{1}$, \\ YASUSHI SUZUKI ${ }^{1}$, HIROAKI TAKASHIMA ${ }^{1}$, and TETSUYA AMANO ${ }^{1}$
}

\author{
${ }^{1}$ Aichi Medical University
}

January 31, 2021

\begin{abstract}
In case of decompensated heart failure related to chemotherapy-induced cardiomyopathy, ivabradine administration could lead to favorable hemodynamics via the increase of stroke volume with reducing sinus heart rate. Assessment of the overlap between the E- and A-waves facilitates understanding the effects of ivabradine in such cases.
\end{abstract}

\section{INTRODUCTION}

The incidence of heart failure (HF) due to chemotherapy-induced cardiomyopathy (CHIC) is increasing and is associated with poor clinical outcomes. Conservative treatment with medications is often difficult, especially in advanced $\mathrm{HF}^{1}{ }^{2}{ }^{2}$. Ivabradine, a specific $\mathrm{I}_{\mathrm{f}}$ channel inhibitor, lowers the sinus heart rate (HR) without affecting myocardial contractility. It is indicated for controlling HR as a second-line medication after the optimal beta-blocker therapy in patients with chronic HF and reduced ejection fraction ${ }^{3,4}$. However, some studies report that ivabradine has a positive effect on the hemodynamics of decompensated $\mathrm{HF}^{5678}$. Here, we report a case of advanced HF related to CHIC refractory to conventional HF therapy successfully treated with ivabradine.

\section{CASE HISTORY / EXAMINATION}

A 61-year-old man with HF related to CHIC, complaining of gradual onset of shortness of breath for 5 days, was admitted to our university hospital for decompensated HF. He had been receiving multiple regimens of chemotherapy, including anthracyclines for the treatment of lymphoma, for 2 years. At the time of his admission, the lymphoma was in remission, but his cardiac function had gradually declined from the normal limit of left ventricular ejection fraction (LVEF) of $60.0 \%$ at baseline. This hospitalization for HF was the third in the previous 10 months.

On admission, his blood pressure was $96 / 64 \mathrm{mmHg}$, pulse rate $118 / \mathrm{min}$, body temperature $36.0{ }^{\circ} \mathrm{C}$, and oxygen saturation $92 \%$ on room air, which increased to $98 \%$ with $4 \mathrm{~L} / \mathrm{min}$ cannula oxygen supply. Physical examination revealed an engorged jugular vein, tachycardia with a grade II systolic murmur over the apex, rales in both lower lung fields, and bilateral edema in the lower extremities.

\section{INVESTIGATIONS AND TREATMENT}

Routine laboratory tests showed an increase in B-type natriuretic peptide (2208 pg/mL), total bilirubin (1.98 $\mathrm{mg} / \mathrm{dL})$, aspartate aminotransferase $(81 \mathrm{U} / \mathrm{L})$, alanine aminotransferase $(75 \mathrm{U} / \mathrm{L})$, blood urea nitrogen $(33.1$ $\mathrm{mg} / \mathrm{dL})$ and creatinine $(1.46 \mathrm{mg} / \mathrm{dL})$. Chest radiography revealed cardiomegaly with pulmonary congestion. Electrocardiography demonstrated sinus tachycardia (HR: 104 /min). Transthoracic echocardiography showed diffuse severe hypokinesis of LVEF 30.8\%, an increased left ventricular end-diastolic diameter of 64.4 $\mathrm{mm}$, and moderate mitral regurgitation (Figure 1). 


\section{OUTCOME AND FOLLOW-UP}

As shown in Figure 2, administration of dobutamine and pimobendane in addition to azosemide and tolvaptan resulted in good diuresis initially, which gradually deteriorated and the patient's HR showed an elevation to $120 / \mathrm{min}$. The subsequent administration of eplerenone and enalapril to manage residual pleural effusion resulted in hemodynamic deterioration with low systolic pressure (approximately $80 \mathrm{mmHg}$ ). Transthoracic echocardiographic examination revealed low stroke volume (SV) and an extreme overlap between the Eand A-waves (Figure 3-left). Administration of oral ivabradine (5 mg twice daily) improved tachycardia and decreased the overlap; the velocity-time integral at the left ventricular outflow tract (LVOT-VTI), SV, and blood pressure increased (Figure 2, and Figure 3-right). His hemodynamics stabilized subsequently, and the pulmonary congestion disappeared, allowing the patient to be weaned from catecholamines. Eventually the LVEF increased to $32.0 \%$ (Figure 4), HF improved, and he was discharged on the 60th day.

This case report has been published with the prior consent of the patient.

\section{DISCUSSION}

The present case shows the favorable hemodynamic effect of ivabradine in a patient with decompensated HF related to CHIC, refractory to conventional medical therapy.

It is reported that the incidence of CHIC due to anthracycline is $9 \%$, with $98 \%$ of the cases developing within 1 year. Anthracycline-induced myocardial damage is irreversible ${ }^{9}$, and the 2 -year survival rate has been reported to be $50 \%$ or less when anthracyclines are the of cause HF. The treatment is often difficult in such cases, where HF is advanced and refractory to inotropes ${ }^{1}$. The use of ivabradine in decompensated HF is off-label. However, due to its unique mechanism of reduction of HR without influencing myocardial contraction, ivabradine does not cause the undesired effect of hypotension, which is a major problem on up-titration of beta-blockers, and might have favorable effects on hemodynamics even in decompensated HF due to CHIC. In some patients in advanced HF or cardiogenic shock, ivabradine was safely used to reduce HR and increase SV without impairing their hemodynamic status ${ }^{5} 7$.

Though the reason ivabradine increases SV in decompensated HF is not fully understood, the mechanisms are suggested to be as follows (Figure 5). Ivabradine causes prolongation of diastolic time, which leads to an increase of LV diastolic filling along with an increase of coronary perfusion, increasing SV via the Franck-Starling mechanism ${ }^{5}, 10,11,12$. Moreover, ivabradine has a positive inotropic effect primarily due to increased sarcoplasmic/endoplasmic reticulum calcium ATPase 2a activity, and does not have a negative inotropic effect, unlike $\beta$-blockers ${ }^{13}$. Afterload reduction based on the reciprocal interaction between HR and effective arterial elastance (Ea) has also been reported ${ }^{10,5,12}$. Ventricular arterial coupling was improved because of the decrease in Ea, resulting in higher SV in patients treated with ivabradine ${ }^{12}$.

How much should the heart rate be reduced by ivabradine? From the viewpoint of echocardiography, monitoring the degree of overlap between the E- and A-waves might be useful as a way to optimize HR (Figure 6). The overlap between E- and A-waves observed at higher sinus HR suggests that LV relaxation is interfered with by atrial systole. And that could result in reduced LV filling volume. Izumida et al. propose a novel formula to estimate ideal HR in patients with HF using echocardiographic parameters, the overlap between the $\mathrm{E}$ - and A-waves, and deceleration time ${ }^{14}$.

In the present case, the HR lowering effect of ivabradine could increase SV, with its attendant favorable hemodynamic consequences. Though medical therapy is often difficult in advanced HF related to CHIC, ivabradine might be effective as a complementary medical option in these cases. Also, assessing the degree of overlap between the E- and A-waves facilitates estimation of the effects of ivabradine.

In conclusion, the administration of ivabradine could lead to improvement of hemodynamic status in decompensated HF related to CHIC via the increase of SV. Assessment of the overlap between the E- and A-waves facilitates receiving the benefits of ivabradine in such cases. Further large-sample verification will be needed to confirm these findings. 


\section{ACKNOWLEDGMENTS}

None.

CONFLICT OF INTEREST

Y.N. receives lecture fees from Ono Yakuhin. The remaining authors have no disclosures to report.

\section{AUTHORSHIP LIST}

YN: Analysis and interpretation of patient's data, conception and design, drafting of the manuscript.

HA: Conception and design, critical feedback and revision of the manuscript.

WS: Revision of the manuscript with a focus on the echocardiographic aspects.

HO: Critical feedback and revision of the manuscript.

YS: Critical feedback and revision of the manuscript.

HT: Critical feedback and revision of the manuscript.

TA: Revision and final approval of the manuscript.

All authors discussed the case and commented on the manuscript at all stages.

\section{ETHICAL APPROVAL}

The Ethics Committee of Aichi Medical University granted an exemption from ethics approval.

\section{REFERENCES}

1. Felker G.M., Thompson R.E., Hare J.M., et al. Underlying causes and long-term survival in patients with initially unexplained cardiomyopathy. $\quad$ N Engl J Med. 2000;342:1077-1084. DOI: $10.1056 /$ NEJM200004133421502

2. Avila M.S., Siqueira S.R.R., Ferreira S.M.A., Bocchi E.A. Prevention and Treatment of ChemotherapyInduced Cardiotoxicity. Methodist Debakey Cardiovasc J. 2019;15:267-273. DOI: 10.14797/mdcj-15-4-267

3. Cowie M.R., Anker S.D., Cleland J.G.F., et al. Improving care for patients with acute heart failure: before, during and after hospitalization. ESC heart failure. 2014;1:110-145. DOI: 10.1161/CIRCOUTCOMES.120.006693

4. Yancy C.W., Jessup M, Bozkurt B, et al. 2017 ACC/AHA/HFSA Focused Update of the 2013 ACCF/AHA Guideline for the Management of Heart Failure: A Report of the American College of Cardiology/American Heart Association Task Force on Clinical Practice Guidelines and the Heart Failure Society of America. J Card Fail. 2017;23:628-651. DOI: 10.1161/CIR.0000000000000509

5. De Ferrari G.M., Mazzuero A, Agnesina L, et al. Favourable effects of heart rate reduction with intravenous administration of ivabradine in patients with advanced heart failure. Eur J Heart Fail.2008;10:550555. DOI: $10.1016 /$ j.ejheart.2008.04.005

6. Franke J, Schmahl D, Lehrke S, et al. Adjuvant Use of Ivabradine in Acute Heart Failure due to Myocarditis. Case Rep Med.2011:203690. DOI: 10.1155/2011/203690

7. Chiu M.H., Howlett J.G., Sharma N.C. Initiation of ivabradine in cardiogenic shock. ESC heart failure. 2019;6:1088-1091. DOI: 10.1002/ehf2.12499

8. Izco M.P., Ramirez-Carracedo R, Navarro I.H., et al. Ivabradine in acute heart failure: Effects on heart rate and hemodynamic parameters in a randomized and controlled swine trial. Cardiol J.2020;27:62-71. DOI: $10.5603 /$ CJ.a2018.0078 
9. Cardinale D, Colombo A, Bacchiani G, et al. Early detection of anthracycline cardiotoxicity and improvement with heart failure therapy. Circulation. 2015;131:1981-1988. DOIi: 10.1161/CIRCULATIONAHA.114.013777

10. Mulder P, Barbier S, Chagraoui A, et al. Long-term heart rate reduction induced by the selective I(f) current inhibitor ivabradine improves left ventricular function and intrinsic myocardial structure in congestive heart failure. Circulation. 2004;109:1674-1679.

DOI: 10.1161/01.CIR.0000118464.48959.1C

11. Custodis F, Schirmer S.H., Baumhakel M, Heusch G, Bohm M, Laufs U. Vascular pathophysiology in response to increased heart rate. J Am Coll Cardiol. 2010;56:1973-1983. DOI: 10.1152/physrev.00042.2012

12. Reil J.C., Tardif J.C., Ford I, et al. Selective heart rate reduction with ivabradine unloads the left ventricle in heart failure patients. J Am Coll Cardiol. 2013;62:1977-1985. DOI: 10.1016/j.jacc.2013.07.027

13. Xie M, Huang H.L., Zhang W.H., et al. Increased sarcoplasmic/endoplasmic reticulum calcium ATPase 2a activity underlies the mechanism of the positive inotropic effect of ivabradine. Exp Physiol. 2020;105:477488. DOI: $10.1113 / \mathrm{EP} 087964$

14. Izumida T, Imamura T, Nakamura M, Fukuda N, Kinugawa K. How to consider target heart rate in patients with systolic heart failure.ESC heart failure. 2020;7:3231-3234. DOI: 10.1002/ejhf.1054

\section{FIGURE LEGENDS}

Figure 1. Long-axis view on echocardiography on admission. Echocardiography showed diffuse severe hypokinesis, increased left ventricular end-diastolic diameter, and moderate mitral regurgitation.

Figure 2. Clinical course. Dobutamine and pimobendane promoted adequate diuresis at the beginning, but gradually result in poor diuresis with HR elevation $(120 \mathrm{bpm})$. After oral ivabradine, the HR decreased and blood pressure increased.

SBP; systolic blood pressure, HR; heart rate, BW; body weight

Figure 3. Echocardiographic changes before (left) and after (right) ivabradine administration. Before ivabradine, echocardiography shows an extreme overlap between the E- and A-waves, low LVOT-VTI, and low SV. After ivabradine, the overlap decreased and LVOT-VTI and SV increased.

HR; heart rate, LVOT-VTI; velocity-time integral at the left ventricular outflow tract, SV; stroke volume

Figure 4. Long-axis view on echocardiography at the time of discharge. Echocardiography showed diffuse severe hypokinesis, but LVEF and mitral regurgitation had improved compared to the time of admission.

Figure 5. The plausible mechanism of an increase of SV by ivabradine. The increased SV by ivabradine is derived from alterations in hemodynamics such as pre-and afterload and myocardial contractility.

HR; heart rate, SV; stroke volume, SERCA2a; sarcoplasmic/endoplasmic reticulum calcium ATPase 2a

Figure 6. HR optimization from the viewpoint of echocardiography.

A: Normal. B: LV diastolic dysfunction prolongs LV relaxation time. C: In higher heart rate, LA systole could interfere with LV complete relaxation. D: Prevention of the E and A waves from overlapping using echocardiographic monitoring might help LV complete relaxation.

LV; left ventricle, LA; left atrial, TMF; trans mitral flow, IRT; isovolumic relaxation time, RF; rapid filling, SF; slow filling, AS; atrial systole, the red line indicates the overlap between the E and A waves 

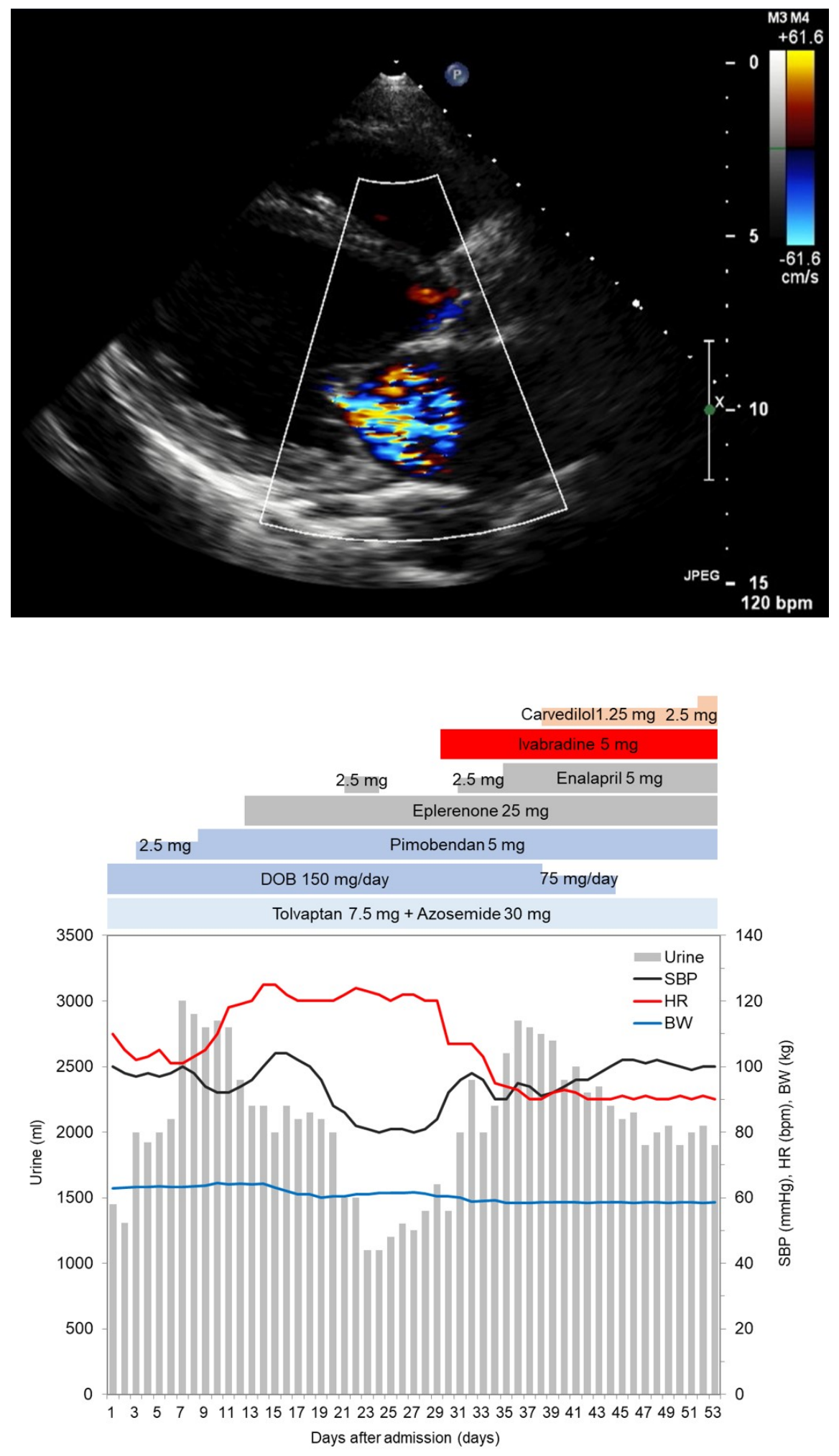

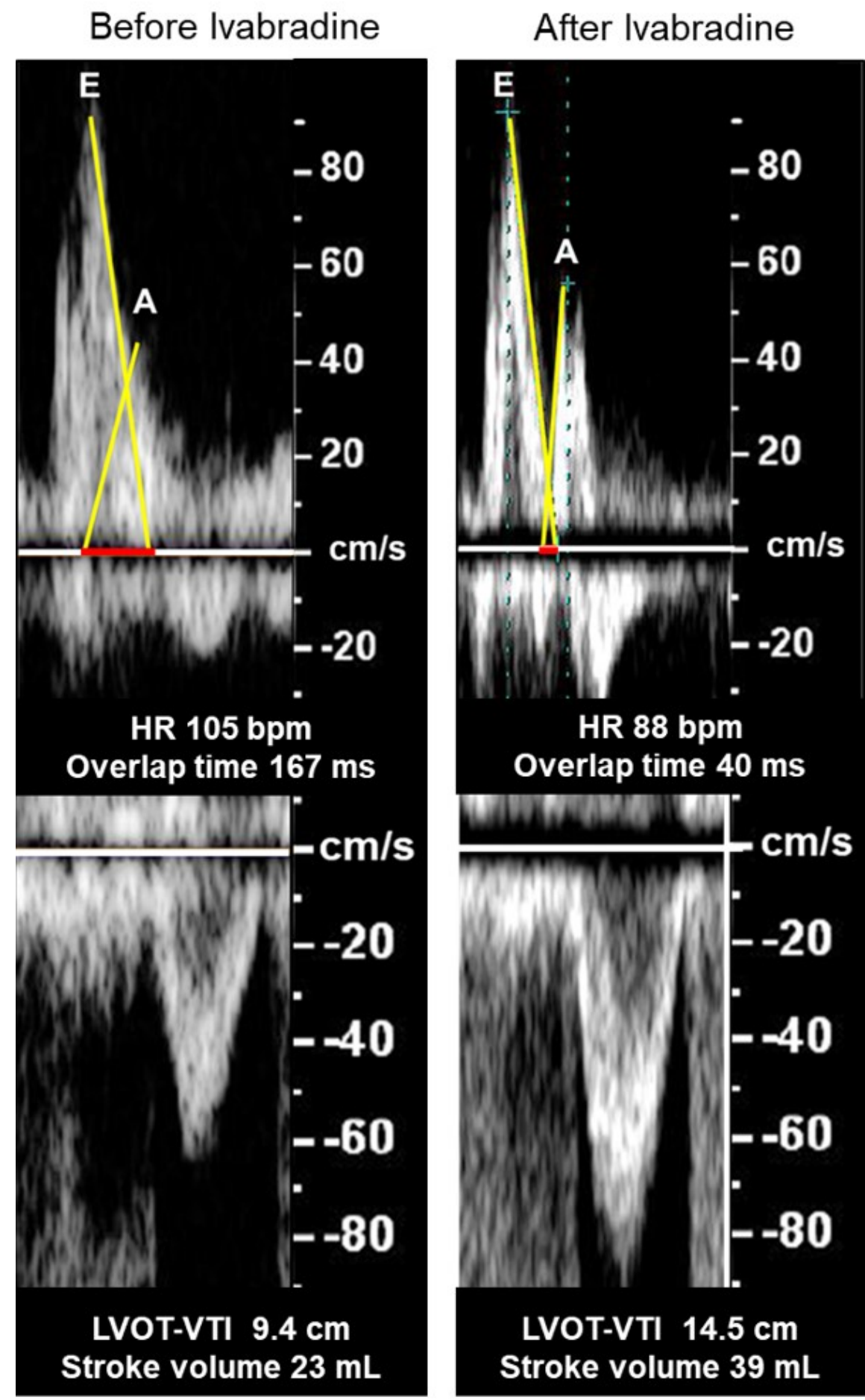

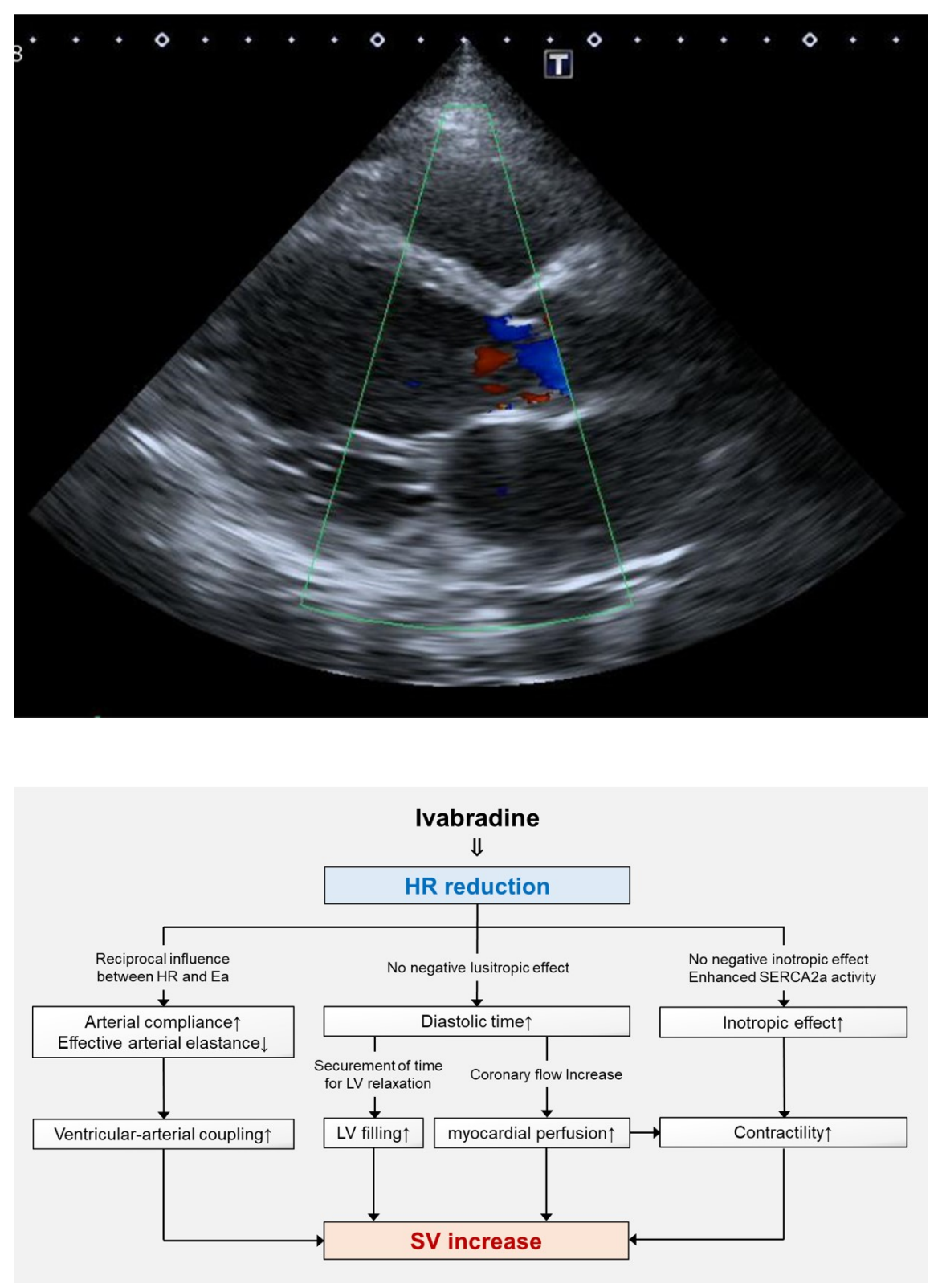
A.

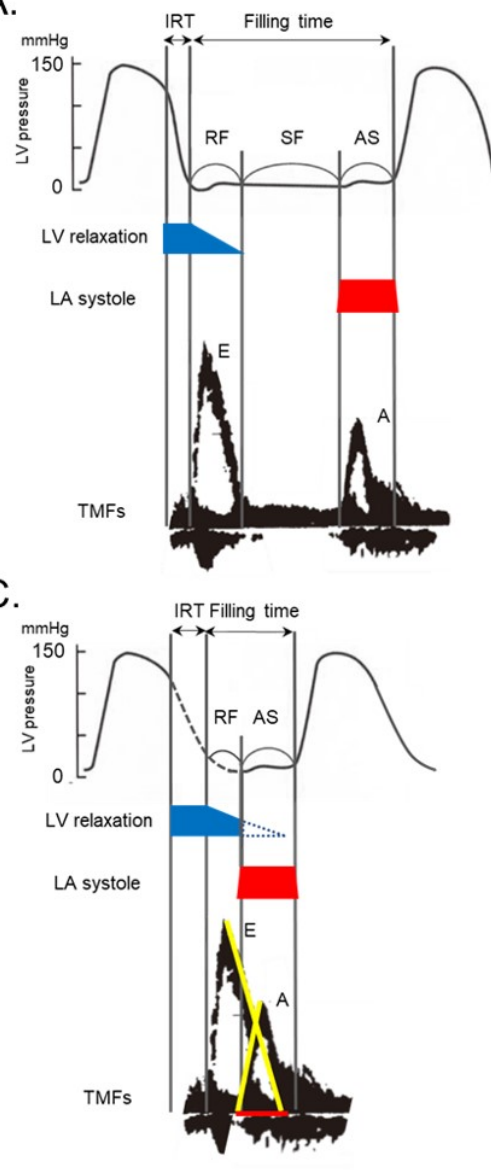

B.

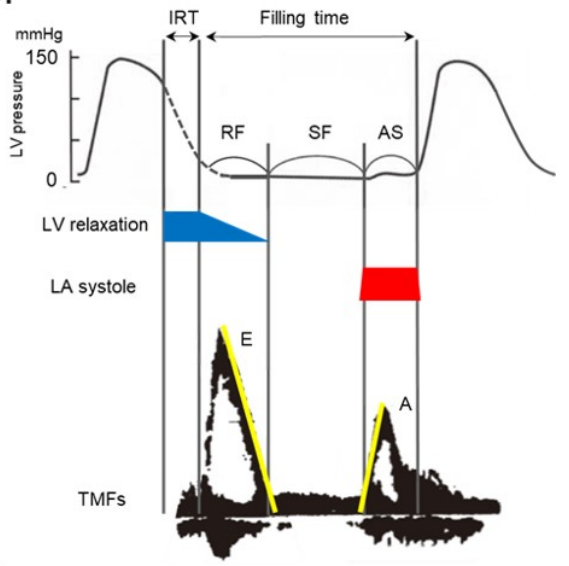

D.

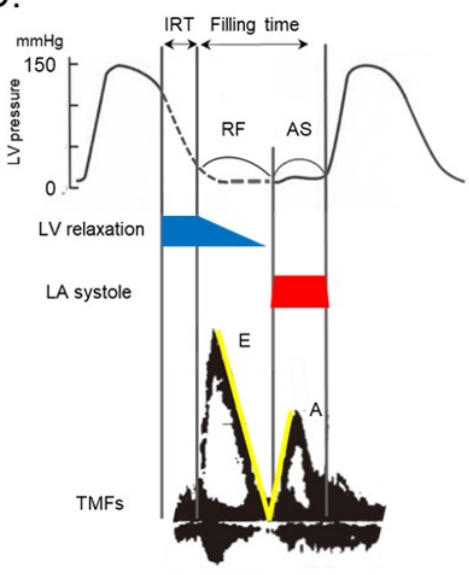

